\title{
Prevalence of ataxia in children
}

\author{
A systematic review
}

Kristin E. Musselman, PT, PhD

Cristina T. Stoyanov, $\mathrm{PhD}$

Rhul Marasigan, BS

Mary E. Jenkins, MD

Jürgen Konczak, PhD

Susanne M. Morton, PT, $\mathrm{PhD}$

Amy J. Bastian, PT, PhD

Correspondence to Dr. Musselman: kristin.musselman@usask.ca

Supplemental data at www.neurology.org

\section{ABSTRACT}

Objective: To estimate the prevalence of childhood ataxia resulting from both genetic and acquired causes.

Methods: A systematic review was conducted following the PRISMA (Preferred Reporting Items for Systematic Reviews and Meta-analyses) statement. Five databases were searched for articles reporting a frequency measure (e.g., prevalence, incidence) of ataxia in children. Included articles were first grouped according to the World Health Organization (WHO) regions and subsequently classified according to etiology (genetic, acquired, or mixed). Each article was assessed for its risk of bias on the domains of sampling, measurement, and analysis. Incidence values were converted to prevalence estimates whenever possible. European prevalence estimates for different etiologies of ataxia were summed to gauge the overall prevalence of childhood ataxia.

Results: One hundred fifteen articles were included in the review. More than $50 \%$ of the data originated from the Europe WHO region. Data from this region also showed the least susceptibility to bias. Little data were available for Africa and Southeast Asia. The prevalence of acquired ataxias was found to vary more greatly across regions than the genetic ataxias. Ataxic cerebral palsy was found to be a significant contributor to the overall prevalence of childhood ataxia across WHO regions. The prevalence of childhood ataxias in Europe was estimated to be $\sim 26 / 100,000$ children and likely reflects a minimum prevalence worldwide.

Conclusions: The findings show that ataxia is a common childhood motor disorder with a higher prevalence than previously assumed. More research concerning the epidemiology, assessment, and treatment of childhood ataxia is warranted. Neurology ${ }^{\circledR} 2014 ; 82: 80-89$

\section{GLOSSARY}

$\mathbf{C I}=$ confidence interval; TAN = tropical ataxic neuropathy; $\mathbf{W H O}=$ World Health Organization .

Ataxia refers to a lack or loss of movement coordination. It is associated with damage or dysfunction of the cerebellum or its afferent and efferent projections. ${ }^{1}$ Ataxia is especially debilitating for children because they are still developing and learning motor competency. ${ }^{2}$ Childhood ataxia can have hereditary causes, such as ataxia telangiectasia, or acquired causes, such as infection or tumor. The duration of ataxia varies greatly: from short, transient episodes, such as postinfectious cerebellar demyelination, ${ }^{3}$ to lifelong conditions, such as Dandy-Walker syndrome.

The prevalence of some ataxia-causing conditions has been reported. However, most reports are focused on a single condition and a specific geographical location, making it difficult to generalize the prevalence estimates. Furthermore, few studies separate the prevalence of ataxia in children vs adults. ${ }^{4-12}$ Given the multitude of ataxia-causing conditions and recognizing that each can be quite rare, the overall prevalence of childhood ataxia is likely underestimated. Yet overall prevalence data are needed to accurately assess the impact and societal cost of childhood ataxia.

The purpose of this article is to systematically review the literature on the prevalence of ataxia in children across World Health Organization (WHO) regions. The prevalence of childhood

From the Department of Neuroscience (K.E.M., A.J.B.), Johns Hopkins School of Medicine, Baltimore, MD; Kennedy Krieger Institute (K.E.M., R.M., A.J.B.), Baltimore, MD; Johns Hopkins Bloomberg School of Public Health (C.T.S.), Baltimore, MD; Department of Clinical Neurological Sciences (M.E.J.), Western University, London, Ontario, Canada; School of Kinesiology (J.K.), University of Minnesota, Minneapolis; and Department of Physical Therapy (S.M.M.), University of Delaware, Newark.

Go to Neurology.org for full disclosures. Funding information and disclosures deemed relevant by the authors, if any, are provided at the end of the article. 
ataxia is estimated by systematically examining prevalence estimates for the many different causes of childhood ataxia.

METHODS Search strategy. A systematic review of the literature was undertaken in consultation with a clinical informationist from Johns Hopkins School of Medicine. The review process was based on the PRISMA (Preferred Reporting Items for Systematic Reviews and Meta-analyses) statement. ${ }^{13}$ The 5 databases searched from the earliest record to April 2, 2012, were PubMed, Web of Science, EMBASE, CINHAL, and Scopus. The search terms (see appendix e-1 on the Neurology ${ }^{\circledR}$ Web site at www. neurology.org) included keywords and controlled vocabulary, if applicable, with 3 concepts being searched: 1) ataxia, 2) measurement of disease frequency (e.g., prevalence, incidence), and 3) childhood. No restrictions were placed on the language or publication date.

Data extraction. Following the literature search, duplicate abstracts were removed using a research management tool (RefWorks, Bethesda, MD). All abstracts were then independently reviewed by 2 authors (K.E.M. and R.M.) to select articles warranting full review. To be selected for full review, the article must have 1) reported on an ataxia-causing condition, 2) included, or been suspected of including, children in the sample, and 3) included, or been suspected of including, some measure of the frequency of ataxia, such as the number of cases, prevalence, incidence, frequency, or percentage of the sample that showed ataxia.

Full paper reviews, which were performed by one author (K.E.M.), first involved determining whether the paper met any exclusion criteria, as follows: 1) conference abstracts, 2) review articles, if the review did not provide an original estimate of disease frequency, 3) articles in which the pediatric and adult data could not be separated, and 4) articles in which the data concerning ataxia could not be separated from data involving another neurologic condition. Reference lists were scanned and relevant references reviewed. To ensure that only articles on ataxia-causing conditions were included, a third author (M.E.J.) independently confirmed the diseases and conditions associated with ataxia. ${ }^{14,15}$

The following information was extracted from included articles: location, time period, method(s) used to identify cases, method(s) used to confirm diagnoses, age range of cases, number of pediatric cases identified, size of the pediatric population, and measure of frequency (e.g., prevalence, incidence, or percentage showing ataxia) with $95 \%$ confidence interval (CI). In the case of transient causes of ataxia, we also attempted to record the average length of time that ataxia was experienced. The majority of included articles were in English, but some were in French, Spanish, German, Portuguese, Croatian, Turkish, Japanese, Icelandic, Danish, and Chinese. For these papers individuals proficient in the relevant language assisted with translation.

Lastly, risk of bias (i.e., the risk that the study over- or underestimated the true prevalence) was assessed. We adopted a domain-based evaluation, in which the likelihood of bias was assessed for different aspects of study design. ${ }^{16,17}$ The following domains were evaluated. ${ }^{18}$

1. Sampling bias was evaluated by how representative the cases were of the intended target population.

2. Measurement bias was evaluated by the method used to identify ataxia or the ataxia-causing condition.

3. Bias in analysis was evaluated by the inclusion of CIs for estimates of prevalence and incidence.

Included articles were given risk ratings of "low," "high," or "unclear" for each of the above 3 domains. ${ }^{17}$
Data analysis. Included articles were classified according to the 6 geographical regions outlined by the WHO (Africa, Americas, Southeast Asia, Europe, Eastern Mediterranean, and Western Pacific) and then further divided according to etiology of ataxiagenetic, acquired, or mixed. Mixed ataxias included conditions believed to be multifactorial in etiology, with both genetics and environment having a role. Examples of mixed ataxias include ataxic cerebral palsy ${ }^{19}$ and pediatric multiple sclerosis. ${ }^{20}$ In the genetic category, autosomal recessive cerebellar ataxias were further categorized into the following 4 groupings: degenerative and progressive ataxias, DNA repair defects, metabolic ataxias, and congenital ataxias. ${ }^{21}$

We used point prevalence (total cases/population at a single point in time) and period prevalence (total cases/population over a specified period of time) as the measures of frequency. For articles that reported incidence or an incidence rate, those measures were converted to prevalence in 1 of 2 ways.

1. Prevalence $=$ incidence if: a) the condition is present from the time of birth, b) the condition cannot be cured, and c) life expectancy with the condition exceeds childhood. Conditions that met these criteria included many of the hereditary ataxias and other childhood-long conditions, such as congenital malformations.

2. Prevalence $=$ incidence $\times$ duration of ataxia ${ }^{22}$ if the ataxia was transient in nature or if the average life expectancy with the condition was shortened such that affected children typically do not reach adulthood. Often an estimate of duration could not be found; thus, we could not calculate prevalence from incidence in these cases.

Some articles did not report prevalence or incidence but instead reported the percentage of the sample that showed ataxia. If the incidence or prevalence of the ataxia-causing condition (e.g., cerebral palsy) could be found for the same geographical region, either in the same article or from another source, then the prevalence of the condition with ataxia could be calculated.

A few articles reported only the number of pediatric cases of an ataxia-causing condition. If the size of the pediatric population in the same geographical location at roughly the same time could be found, prevalence was calculated by dividing the number of pediatric cases by the total pediatric population.

Prevalence estimates are reported with a 95\% CI. If the CI was not reported in the article, it was calculated using the Jeffreys method $^{23}$ whenever possible. Prevalence estimates for genetic, acquired, and mixed ataxias were displayed in forest plots. Only prevalence values with a similar denominator (i.e., rate/100,000 children aged $\sim 0-19$ years) were included in the plots. Since the methods used to identify and diagnose cases of childhood ataxia varied greatly across articles, it was not possible to combine data across articles as is done in a meta-analysis. Furthermore, the scarcity of prevalence data available for all WHO regions except Europe limited the analysis. For the European region we reported the median and range of prevalence estimates for each ataxiacausing condition and summed the median values to gauge the total prevalence of childhood ataxia in Europe.

RESULTS The results from the search and screening are outlined in figure 1 . The search returned almost 6,000 abstracts, and of the 355 articles reviewed in full, 115 were included. A summary of the included articles, as well as an assessment of each study's risk of bias, can be found in appendix e-2. The likelihood of bias varied with WHO region, with the European studies being the least susceptible to bias on average. Likewise, we 


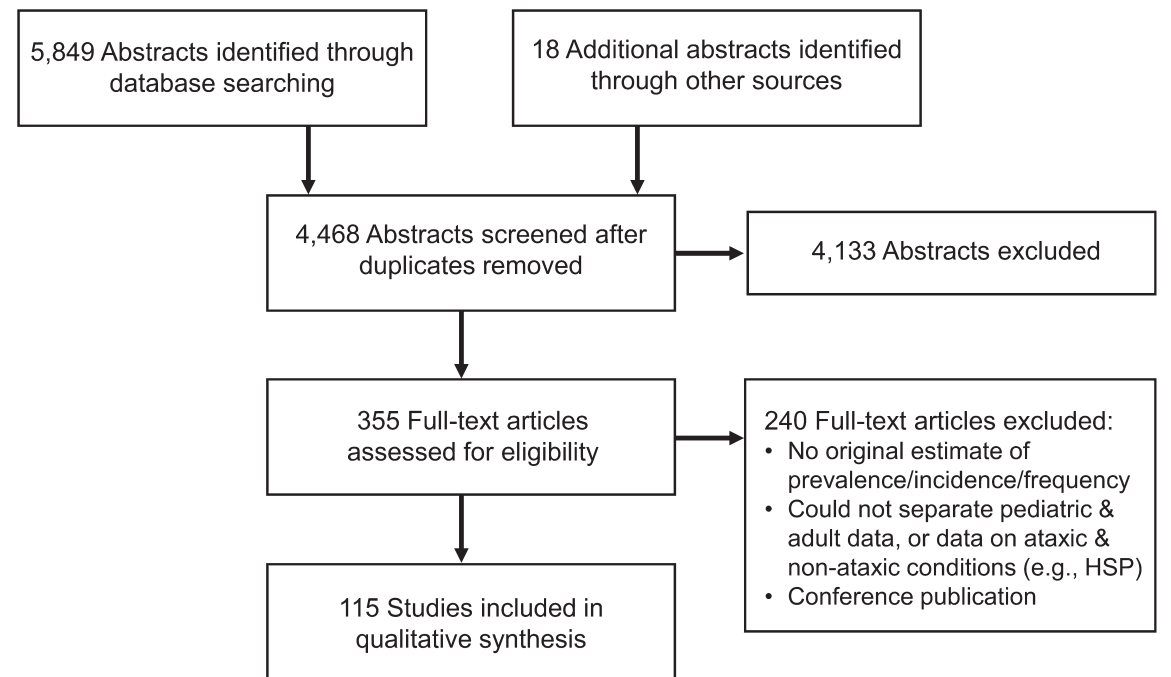

Common reasons for exclusion of articles are listed in the bottom right box. HSP = hereditary spastic paraplegia

found childhood ataxia-the causes, reporting, and prevalence-to vary considerably by region.

Europe. The most represented WHO region in the data was Europe; $52 \%$ of the included studies were conducted in European countries (figure 2). More than $80 \%$ of the European articles were from Western Europe. While few articles had publication dates prior to 1990 , prevalence dates and periods covered many decades. Data were found for all 4 categories of autosomal recessive cerebellar ataxia. ${ }^{21}$ One article reported the prevalence of autosomal dominant cerebellar ataxia in children. ${ }^{11}$ Sixteen articles looked at the frequency of ataxic cerebral palsy. Data were also available for Rett syndrome, hemophagocytic lymphohistiocytosis, Dandy-Walker syndrome, cerebellar hypoplasia, intracerebral hemorrhage, celiac disease, pediatric multiple sclerosis, varicella, tickborne encephalitis, acute disseminated encephalomyelitis, sclerosing panencephalitis secondary to measles, the measles-mumps-rubella vaccine, tumors, and ataxia caused by epilepsy medication.

In Europe, population-based studies were the norm; typically researchers sought to identify all cases

Figure 2 Dot distribution map made using ArcGIS (Ersi, Redlands, CA)

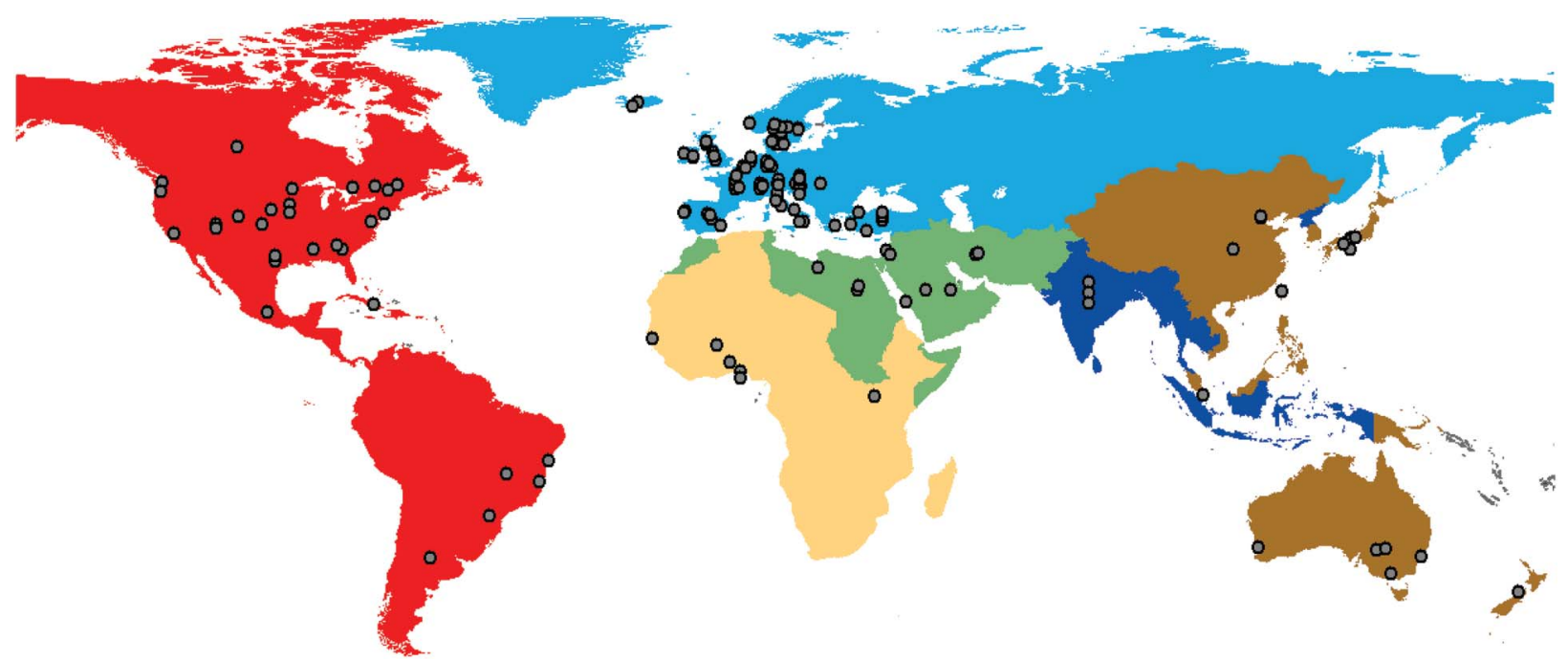

Each color represents a different World Health Organization region: Europe (light blue), Americas (red), Eastern Mediterranean (green), Western Pacific (brown), Africa (yellow), Southeast Asia (dark blue). Gray dots indicate the location of articles returned in the search. If an article reported data for an entire country, the dot is placed near the middle of the country. One article may be represented by more than one dot if the article reported data from more than one country. 
by accessing numerous resources or relying on nationwide registries or screening programs. Mandatory newborn screening programs exist in several European countries, and there are also some far-reaching organizations for surveillance of neurologic disorders; thus, there was a wealth of population-based data on the incidence of various ataxias. Often ataxia was identified through a neurologic exam by a physician; however, sometimes its presence was inferred through medical records or questionnaires, which increased the likelihood of bias. Most studies reported a CI for the estimate of frequency or the data needed to calculate the interval.

Americas. The Americas accounted for $\sim 23 \%$ of included articles, with more than half coming from the United States (figure 2). There was a variety of prevalence dates and periods (1941-2009). Data, albeit sparse compared with Europe, were found for all but one category of autosomal recessive cerebellar ataxia-the congenital ataxias. ${ }^{21}$ The prevalence of autosomal dominant cerebellar ataxia in children was reported for Holguín, Cuba; however, this area has the highest prevalence of spinocerebellar ataxias in the world, ${ }^{9}$ so its prevalence is not representative of the rest of the Americas. The prevalence of autosomal recessive spastic ataxia of Charlevoix-Saguenay and of Leigh syndrome was reported for children in regions of Quebec, Canada, where there is a much higher prevalence of both diseases compared with the rest of the world, due to consanguinity. ${ }^{24,25}$ Only 2 studies, also from Quebec, reported on the frequency of ataxic cerebral palsy. ${ }^{26,27}$ There were also data for Kawasaki disease, alternating hemiplegia of childhood, and basilar-type migraine. There were 5 to 6 articles found each for drug-induced ataxias (side effect or poisoning) and tumors. The frequency of drug-induced ataxia varied with the drug; prevalence estimates for the drug-induced ataxias were not provided. Likewise, the number of children experiencing ataxia as result of a tumor varied with the location of the tumor. Data were also found for acute disseminated encephalomyelitis, bacterial neonatal meningitis, and varicella.

Samples of convenience were frequently used in the studies from the Americas, for example, reports of calls to poison control centers or retrospective chart reviews. Thus, the risk of sampling bias was high. The risk of measurement bias was more variable, with some studies conducted prospectively and requiring neurologic exams (smaller likelihood of bias) and others relying on retrospective review of medical records (greater likelihood of bias). A CI surrounding the prevalence/frequency estimate was reported or calculated for most articles; thus, the bias related to analysis was low.
Western Pacific. In the Western Pacific region, Australia and Japan were the most represented countries, followed by China. Prevalence periods spanned from 1970 to 2008. Data were found for the prevalence of several metabolic ataxias; however, these were the only autosomal recessive ataxias studied. Data were also found on ataxic cerebral palsy, Rett syndrome, alternating hemiplegia of childhood, hemophagocytic lymphohistiocytosis, basilar-type migraine, varicella, meningitis, and ataxia resulting from petrol sniffing.

The risk of bias varied across studies. Some studies used robust sampling methods, such as national registries, whereas others relied on samples of convenience. Likewise, ataxia may have been identified through direct contact and evaluation of the cases or through retrospective chart review. CIs were calculated for most studies; thus, the risk of bias with respect to analysis was low.

Eastern Mediterranean. In the Eastern Mediterranean region, 9 articles were included and covered Saudi Arabia, Egypt, Iran, Libya, and Jordan. Prevalence periods were relatively recent, with the earliest reported year being 1985 . Friedreich ataxia and ataxia telangiectasia were the only autosomal recessive ataxias for which prevalence estimates were reported. Data were also found for Dandy-Walker syndrome, ataxic cerebral palsy, and pediatric multiple sclerosis.

Overall, the risk of sampling bias was deemed high, as samples of convenience and retrospective reviews of medical records were frequently employed. The risk of bias related to measurement and analysis was lower, as many studies confirmed diagnoses through clinical exams and/or imaging and reported sufficient data to calculate CIs.

Africa. We found data on only 2 ataxia-causing conditions in Africa. Both were acquired ataxias that are somewhat unique to this WHO region. Tropical ataxic neuropathy (TAN) is a polyneuropathy that may result from consumption of cassava, a cyanideproducing food. ${ }^{10}$ The prevalence of TAN in several Nigerian communities is extremely high, $\sim 260$ to $570 / 100,000$ children aged 10 to 19 years. ${ }^{8,10}$ These prevalence estimates were obtained by meticulous case finding and diagnosis in the community (e.g., door-to-door screening of the entire local population and follow-up neurologic exams for all suspected cases). ${ }^{8,10}$ Thus, the risk of bias was deemed low.

Data were also found for malaria-induced ataxia. Malaria is very prevalent in children in some areas of Africa. For example, a prevalence of 49,000/ 100,000 children has been reported. ${ }^{28}$ A small percentage of children with malaria experience ataxia $(0.2 \%-2.8 \%) .^{29-31}$ The studies concerning malaria involved samples of convenience, so the risk of sampling bias was high. Two of the 3 articles, however, 
Figure 3 Prevalence of genetic ataxias in children (rate/100,000 and 95\% confidence interval)

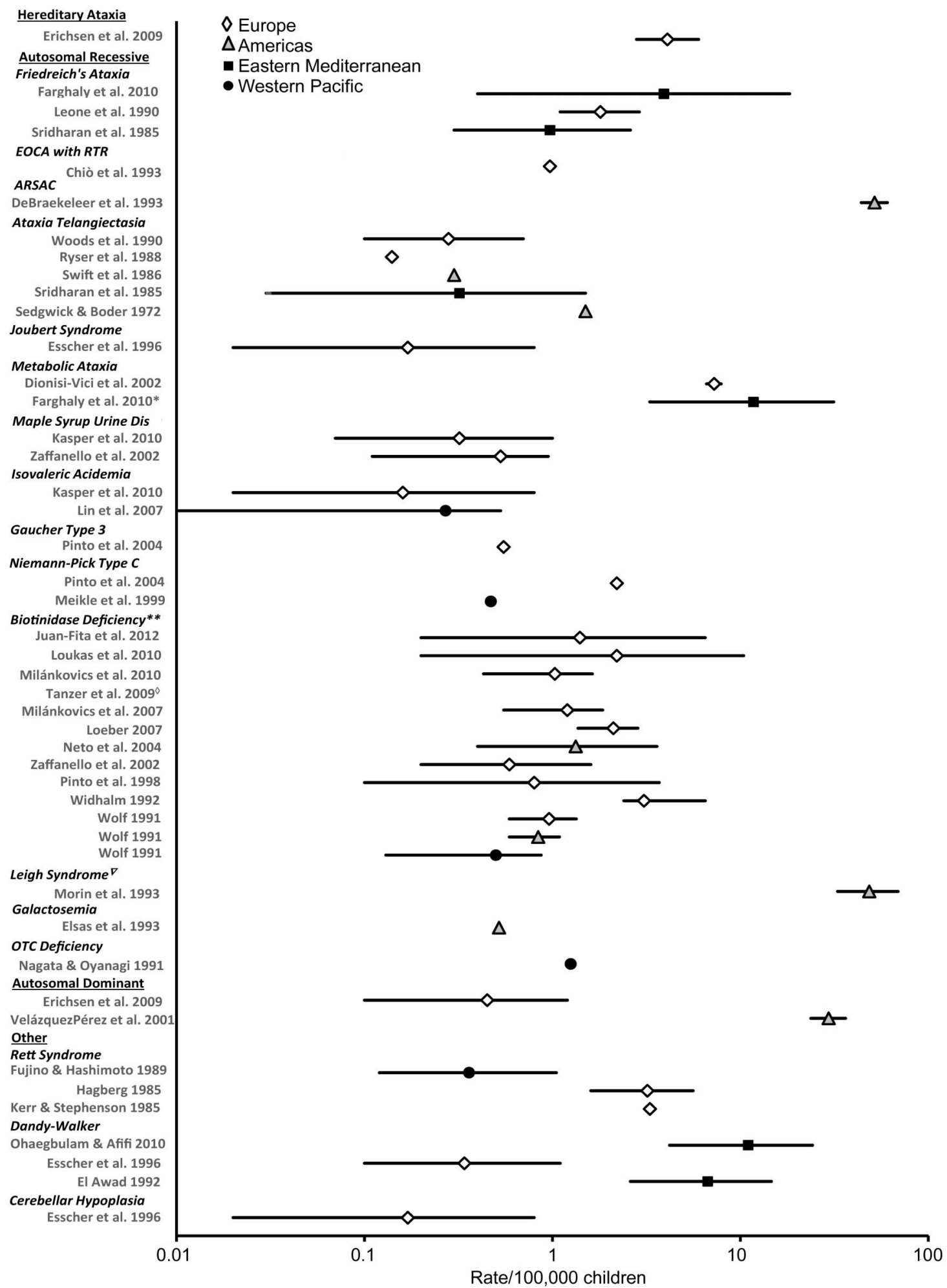

Each symbol represents the prevalence estimate from a specific World Health Organization (WHO) region. Different symbols represent different WHO regions. Prevalence rates are plotted on a logarithmic scale because of the large range of rates reported. *Study of progressive ataxia in which authors stated

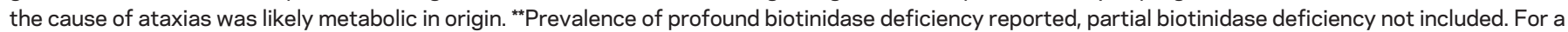
complete list of the metabolic ataxias included in the results of Dionisi-Vici et al. (2002), see appendix e-2. $\diamond$ Prevalence of 0/100,000 reported in this article. ${ }^{\nabla}$ Mitochondrial disorder. For citations, see appendix e-2. ARSAC = autosomal recessive spastic ataxia of Charlevoix-Saguenay; EOCA with RTR = earlyonset cerebellar ataxia with retained tendon reflexes; OTC $=$ ornithine transcarbamylase. 


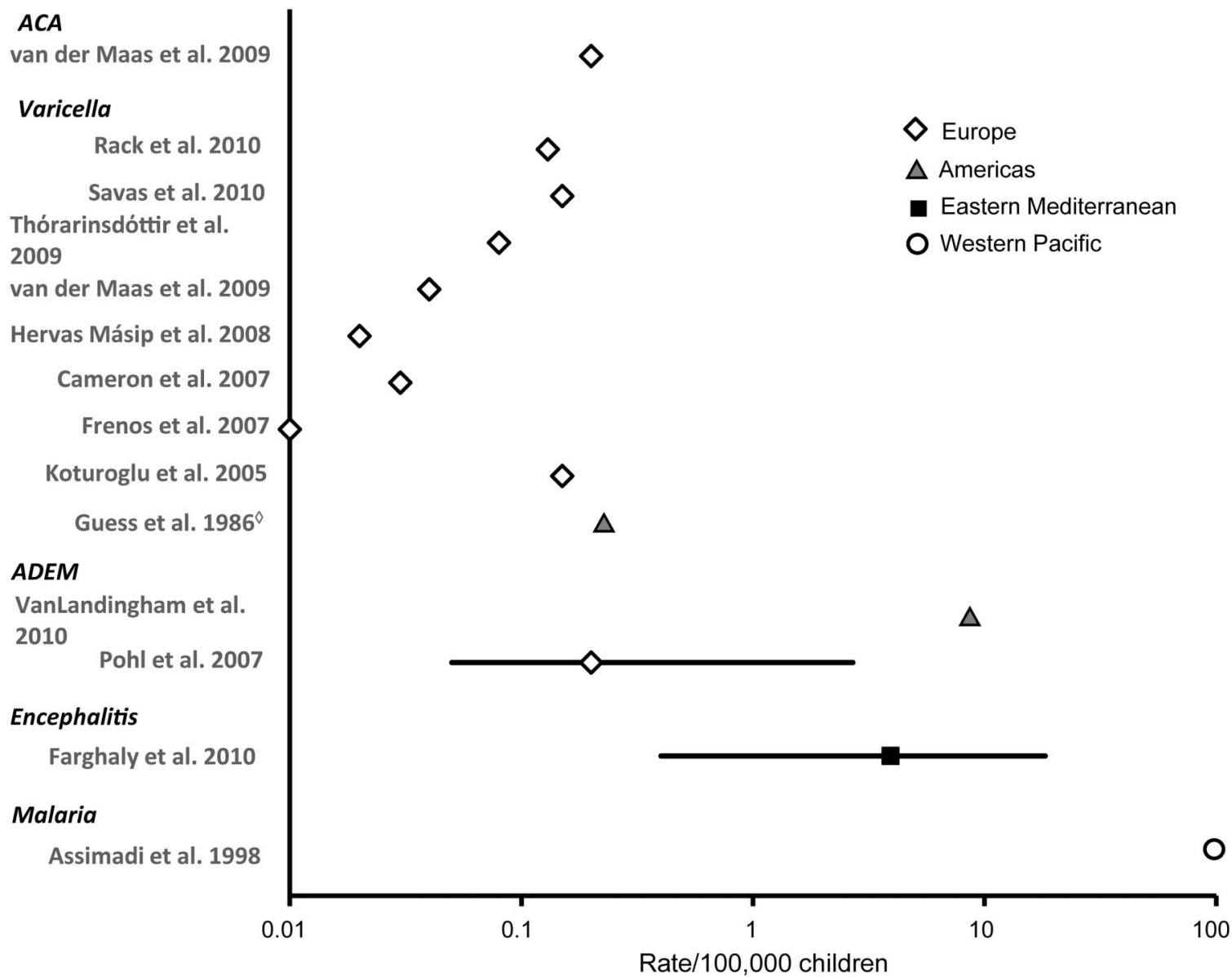

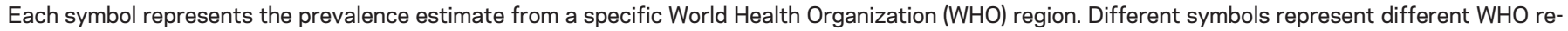

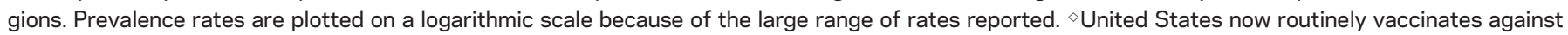
varicella. For citations, see appendix e-2. ACA = acute cerebellar ataxia; ADEM = acute disseminated encephalomyelitis.

used WHO criteria for the diagnosis of malaria and neurologic exams to identify ataxia; thus, the risk of measurement bias was deemed more favorable. CIs could be calculated for all data from Africa.

Southeast Asia. The least amount of data came from Southeast Asia. Only 3 studies, all conducted in India, were included from this WHO region. The articles reported on leukodystrophy, ${ }^{32}$ somatoform disorders, ${ }^{33}$ and acute disseminated encephalomyelitis. ${ }^{34}$ All involved samples of convenience. Thus the risk of sampling bias was high, yet the risk of bias from measurement and analysis was considered low.

Prevalence of genetic, acquired, and mixed ataxias. Forest plots of the prevalence of genetic, acquired, and mixed ataxias are presented in figures 3-5, respectively. Articles that reported the prevalence over the whole range of childhood (i.e., rate per 100,000 children aged $\sim 0$ to 19 years) are represented in the plots. For example, the prevalence of TAN, which was reported as rate per 100,000 children aged 10 to 19 years, was not included.
Overall prevalence of childhood ataxia in Europe. To gauge the overall prevalence of ataxia in children, data from Europe were used. Data from this region were chosen since they 1) covered the greatest number of ataxia-causing conditions, 2) reported prevalence estimates, as opposed to simply a percentage, and 3) were deemed the least susceptible to bias of all the WHO regions. The overall prevalence of ataxia was estimated to be $\sim 26 / 100,000$ children, with genetic and mixed ataxias combined accounting for most of that figure (table).

DISCUSSION Here we show that childhood ataxia is more common than might be interpreted based on isolated studies of specific ataxia-causing conditions. Our estimate of the prevalence of childhood ataxia is $26 / 100,000$ children. While childhood ataxia is rare relative to childhood conditions like cerebral palsy $(211 / 100,000 \text { children })^{35}$ and autism spectrum disorders $\left(620 / 100,000\right.$ children), ${ }^{36}$ it is more prevalent than conditions like hereditary spastic paraparesis $(3.8 / 100,000) .{ }^{11}$ Our prevalence estimate is based on 


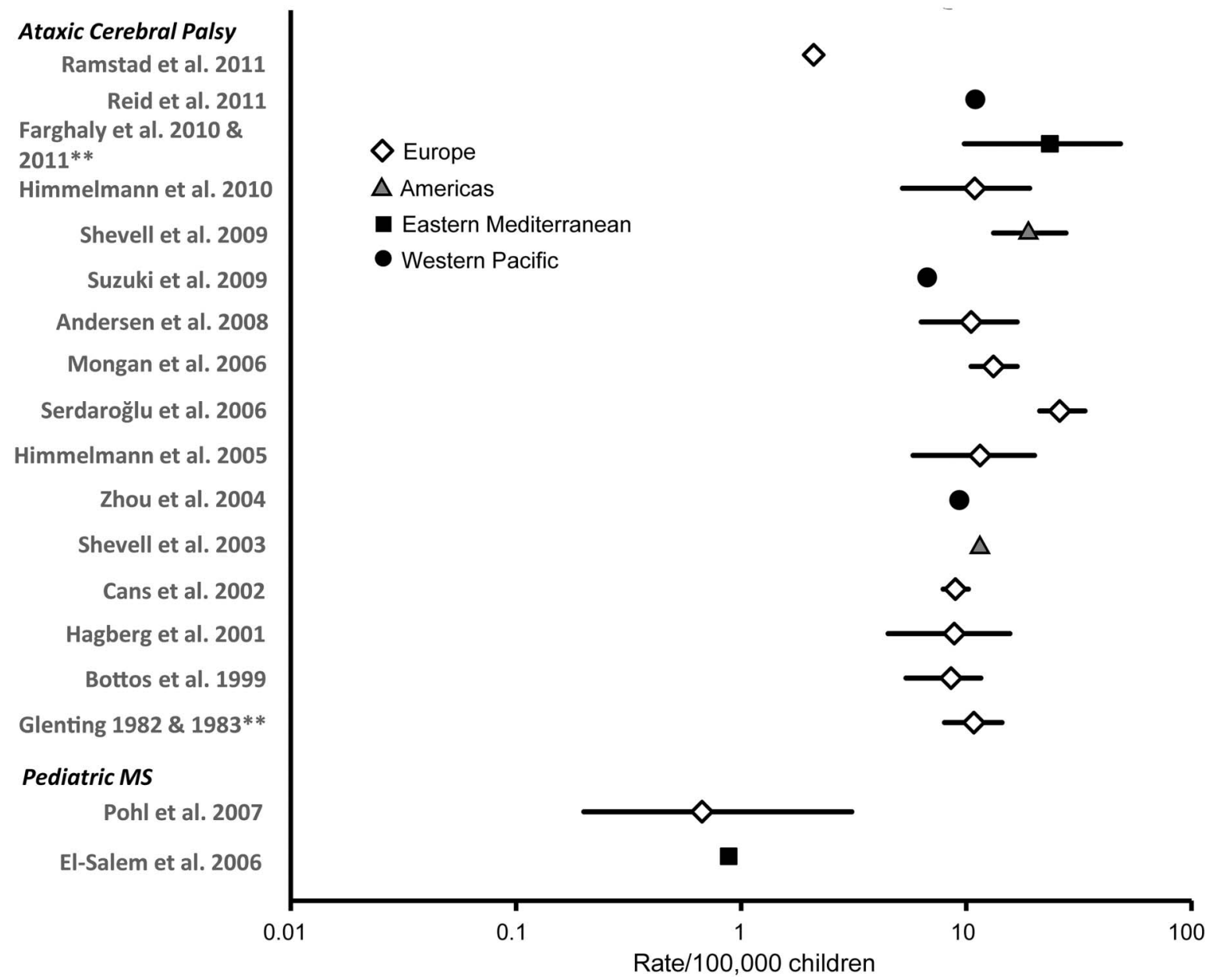

Each symbol represents the prevalence estimate from a specific World Health Organization (WHO) region. Different symbols represent different WHO regions. Prevalence rates are plotted on a logarithmic scale because of the large range of rates reported. **Two articles report on same dataset. For citations, see appendix e-2. MS = multiple sclerosis.

data from Europe, which has a comprehensive pediatric health care system. Knowing that a similar system is not necessarily found in the other WHO regions, the true worldwide prevalence of childhood ataxia is likely higher.

Regional differences. The causes of ataxia varied by WHO region due to regional differences in genetic background, environmental risks, public health policies, and resources. Estimates of genetic conditions were higher in countries with known consanguinity. For example, the prevalence of early-onset cerebellar ataxia with retained tendon reflexes was lower in northwestern Italy $(0.97 / 100,000 \text { children })^{7}$ compared with the Al-Kharga District in Egypt (3.92/ 100,000 children). ${ }^{12}$ Ataxias attributable to environmental causes also varied by region. $\mathrm{TAN}^{8,10}$ and ataxia as a complication of malaria ${ }^{29-31}$ were only reported from Africa. We speculate that public health policies led to some differences between regions. For example, the prevalence of ataxia secondary to varicella will depend on a country's requirements for varicella vaccination. Finally, data from newborn screening programs were only available from more developed countries, possibly reflecting differences in resources available for tracking human health.

Strengths and limitations of review. Estimating the prevalence of ataxia, which is characterized by such a large and heterogeneous group of etiologies, is challenging. The first strength of this review is that it identifies gaps in our current knowledge about the prevalence of childhood ataxia and highlights areas in need of research. Second, we collected all available data from all WHO regions. Identifying regional differences in the prevalence and causes of childhood ataxia will guide future region-specific work. Third, this review can serve as a model for future studies investigating the epidemiology of a clinical disorder that has a number of varied etiologies. Finally, we would like to note that without initiatives to systematically record the presence of ataxia regardless of 
Table Summary of childhood ataxia in Europe

\begin{tabular}{lll} 
& $\begin{array}{l}\text { No. of } \\
\text { articles }\end{array}$ & $\begin{array}{l}\text { Median prevalence } \\
\text { (range) per 100,000 children }\end{array}$ \\
\hline Friedreich ataxia & 1 & 1.8 \\
\hline Early-onset CA with RTR & 1 & 0.97 \\
\hline Ataxia telangiectasia & 2 & $0.21(0.14-0.28)$ \\
Joubert syndrome & 1 & 0.17 \\
Metabolic ataxia & 1 & 7.25 \\
Autosomal dominant CA & 1 & 0.45 \\
Rett syndrome & 2 & $3.25(3.2-3.3)$ \\
\hline Dandy-Walker syndrome & 1 & 0.34 \\
Cerebellar hypoplasia & 1 & 0.17 \\
Sum genetic etiology & & 14.61 \\
Varicella & 8 & $0.06(0.01-0.15)$ \\
\hline ADEM & 1 & 0.2 \\
\hline Sum acquired etiology & & 0.26 \\
Ataxic cerebral palsy & 10 & $10.65(2.1-25.96)$ \\
\hline Pediatric MS & 1 & 0.67 \\
Sum mixed etiology & & 11.32 \\
Sum genetic, acquired, \& mixed & & 26.19 \\
\hline
\end{tabular}

Abbreviations: $\mathrm{ADEM}=$ acute disseminated encephalomyelitis; $\mathrm{CA}=$ cerebellar ataxia; MS = multiple sclerosis; RTR = retained tendon reflexes.

etiology, a well-designed systematic review is the most effective method for obtaining this information in a timely and cost-effective manner.

There are a few limitations of our review to note. First, there is considerable variability in the type and quality of data from different WHO regions, which limits the generalizability of our findings. We did not include regional search engines, hence many of the articles came from European or North American journals. This may have limited the number of articles from non-Western countries. Furthermore, we were unable find data on all the different ataxiacausing conditions. For example, prevalence estimates for ataxia with oculomotor apraxia were not retrieved from any WHO region. Second, we did not discriminate between the methods used to confirm the presence of ataxia, as we wanted to err on the side of inclusiveness. The likelihood of bias may have been less had we excluded studies that ascertained cases through questionnaires or chart reviews. Third, we included conditions that cause ataxia in most cases; however, it is possible that some of the cases included in a prevalence estimate did not experience ataxia. Finally, we did not exclude any articles based on the prevalence period, so our review spans $\sim 60$ to 70 years. The prevalence of some conditions may change over time, although the prevalence of cerebral palsy, the most common single cause of childhood ataxia, has been stable since at least the 1980s. ${ }^{35}$
Likewise, diagnostic methods change over time, and older methods may not be as sensitive, reliable, or accurate as current methods, resulting in changing prevalence estimates across time. ${ }^{11}$ The likelihood of bias may have been reduced by limiting our review to studies with recent prevalence periods; however, this would have significantly reduced the amount of data included. It is also noteworthy that classification systems and names of conditions change over time, both of which may have made the data difficult to interpret. For example, as disease-causing genes are identified, nonspecific terms like early-onset cerebellar ataxia with retained tendon reflexes are used less frequently.

Recommendations for the future. This review suggests that childhood ataxia is not an insignificant problem and that it is experienced across the globe. Our findings also suggest a number of shortcomings concerning the study of childhood ataxia and lead to the following recommendations. First, regional and global initiatives are needed to undertake epidemiologic studies of ataxia in children. Data from less developed regions are especially needed. Such initiatives exist for other childhood conditions, such as the International Autism Epidemiology Network. ${ }^{36}$ Second, standard criteria for the diagnosis of ataxia should be established. This will facilitate the aggregation and comparison of data across studies and geographical regions. Third, more research concerning the measurement and treatment of childhood ataxia is needed. There are few reports specific to children regarding the measurement ${ }^{37}$ and treatment of ataxia (pharmacologic ${ }^{38-42}$ and nonpharmacologic ${ }^{43-45}$ ). This may reflect the fact that the overall prevalence and importance of childhood ataxia has likely been underestimated in the past, despite the fact that ataxia is associated with significant economic cost and decreased quality of life. ${ }^{46}$ We hope the findings of this review increase awareness of childhood ataxia and motivate further study on this serious childhood affliction.

\section{AUTHOR CONTRIBUTIONS}

KE Musselman: study concept and design, acquisition of data, analysis of data, interpretation of data, writing of manuscript. CT Stoyanov: analysis of data, interpretation of data, revising manuscript. R Marasigan: analysis of data, revising manuscript. ME Jenkins: study concept and design, interpretation of data, revising manuscript. J Konczak: study concept and design, revising manuscript. SM Morton: study concept and design, revising manuscript. AJ Bastian: study concept and design, interpretation of data, writing of manuscript.

\section{ACKNOWLEDGMENT}

The authors thank Victoria Goode, MLIS, for assisting with the literature search and Junichi Tajino, Gisli Kort Kristofersson, Jane Lee-Wu, Kerry Wu, Bill Hoard, and Alex Vasquez for assisting with translation.

\section{STUDY FUNDING}

The National Institute of Neurological Disorders and Stroke (U13 NS061384) and Canadian Institutes of Health Research (K.E.M) funded this research. 


\section{DISCLOSURE}

The authors report no disclosures relevant to the manuscript. Go to Neurology.org for full disclosures.

Received April 24, 2013. Accepted in final form November 4, 2013

\section{REFERENCES}

1. Manto MU. Clinical signs of cerebellar disorders. In: Manto MU, Pandolfo M, editors. The Cerebellum and Its Disorders. Cambridge: Cambridge University Press 2002:97-120.

2. Konczak J, Timmann D. The effect of damage to the cerebellum on sensorimotor and cognitive function in children and adolescents. Neurosci Biobehav Rev 2007;31 1101-1113.

3. Ryan MM, Engle EC. Acute ataxia in childhood. J Child Neurol 2003;18:309-316.

4. Leone M, Brignolio F, Rosso MG, et al. Friedreich's ataxia: a descriptive epidemiological study in an Italian population. Clin Genet 1990;38:161-169.

5. Werdelin L, Keiding N. Hereditary ataxias: epidemiological aspects. Neuroepidemiology 1990;9:321-331.

6. Woods CG, Bundey SE, Taylor AM. Unusual features in the inheritance of ataxia telangiectasia. Hum Genet 1990; 84:555-562.

7. Chiò A, Orsi L, Mortara P, Schiffer D. Early onset cerebellar ataxia with retained tendon reflexes: prevalence and gene frequency in an Italian population. Clin Genet 1993; 43:207-211.

8. Oluwole OS, Onabolu AO, Link H, Rosling $\mathrm{H}$. Persistence of tropical ataxic neuropathy in a Nigerian community. J Neurol Neurosurg Psychiatry 2000;69: 96-101.

9. Velázquez-Pérez L, Santos FN, García R, Paneque HM, Hechavarría PR. Epidemiology of Cuban hereditary ataxia. Rev Neurol 2001;32:606-611.

10. Oluwole OS, Onabolu AO, Cotgreave IA, Rosling H, Persson A, Link H. Low prevalence of ataxic polyneuropathy in a community with high exposure to cyanide from cassava foods. J Neurol 2002;249:1034-1040.

11. Erichsen AK, Koht J, Stray-Pedersen A, Abdelnoor M, Tallaksen CM. Prevalence of hereditary ataxia and spastic paraplegia in southeast Norway: a population-based study. Brain 2009;132:1577-1588.

12. Farghaly WM, El-Tallawy HN, Rageh TA, Shehata GA, Metwally NA, Abo-Elfetoh NM. Epidemiology of cerebellar ataxia in Al-Kharga district-New Valley (Egypt). Neuroepidemiology 2010;47:527-532.

13. Moher D, Liberati A, Tetzlaff J, Altman DG. Preferred reporting items for systematic reviews and meta-analyses: the PRISMA statement. Ann Intern Med 2009;151: 264-269.

14. Menkes JH, Sarnat HB, Maria BL, editors. Child Neurology. 7th ed. Philadelphia: Lippincott Williams and Wilkins; 2006.

15. Online Mendelian Inheritance in Man. Available at: http://www.omim.org/. Accessed November 23, 2012.

16. Liberati A, Altman DG, Tetzlaff J, et al. The PRISMA statement for reporting systematic reviews and metaanalyses of studies that evaluate health care interventions: explanation and elaboration. Ann Intern Med 2009;151: W65-W94.

17. Higgins JPT, Altman DG, Sterne JAC. Chapter 8: Assessing risk of bias in included studies. In: Higgins JPT,
Green S, editors. Cochrane Handbook for Systematic Reviews of Interventions, Version 5.1.0. The Cochrane Collaboration; Updated March 2011. Available at: www. cochrane-handbook.org. Accessed Feb 7, 2013.

18. Boyle MH. Guidelines for evaluating prevalence studies. Evid Based Ment Health 1998;1:37-39.

19. Marret S, Vanhulle C, Laquerriere A. Pathophysiology of cerebral palsy. Handb Clin Neurol 2013;111:169-176.

20. Van Haren K, Waubant E. Therapeutic advances in pediatric multiple sclerosis. J Pediatr 2013;163:631-637.

21. Palau F, Espinos C. Autosomal recessive cerebellar ataxias. Orphanet J Rare Dis 2006;1:47-66.

22. Freeman J, Hutchison GB. Prevalence, incidence and duration. Am J Epidemiol 1980;112:707-723.

23. Brown LD, Cai TT, DasGupta A. Interval estimation for a binomial proportion. Stat Sci 2001;16:101-133.

24. Morin C, Mitchell G, Larochelle J, et al. Clinical, metabolic, and genetic aspects of cytochrome $\mathrm{C}$ oxidase deficiency in Saguenay-Lac-Saint-Jean. Am J Hum Genet 1993;53:488-496.

25. De Braekeleer M, Giasson F, Mathieu J, Roy M, Bouchard JP, Morgan K. Genetic epidemiology of autosomal recessive spastic ataxia of Charlevoix-Saguenay in Northeastern Quebec. Genet Epidemiol 1993;10:17-25.

26. Shevell MI, Majnemer A, Morin I. Etiologic yield of cerebral palsy: a contemporary case series. Pediatr Neurol 2003;28:352-359.

27. Shevell MI, Dagenais L, Hall N; REPACQ Consortium. Comorbidities in cerebral palsy and their relationship to neurologic subtype and GMFCS level. Neurology 2009; 72:2090-2096

28. Nahum A, Erhart A, Mavé A, et al. Malaria incidence and prevalence among children living in a peri-urban area on the coast of benin, west Africa: a longitudinal study. Am J Trop Med Hyg 2010;83:465-473.

29. van Hensbroek MB, Palmer A, Jaffar S, Schneider G, Kwiatkowski D. Residual neurologic sequelae after childhood cerebral malaria. J Pediatr 1997;131:125-129.

30. Assimadi JK, Gbadoé AD, Atakouma DY, et al. Severe malaria in children in Togo. Arch Pediatr 1998;5: 1310-1315.

31. Opoka RO, Bangirana P, Boivin MJ, John CC, Byarugaba J. Seizure activity and neurological sequelae in Ugandan children who have survived an episode of cerebral malaria. Afr Health Sci 2009;9:75-81.

32. Singhal BS, Gursahani RD, Udani VP, Biniwale AA. Megalencephalic leukodystrophy in an Asian Indian ethnic group. Pediatr Neurol 1996;14:291-296.

33. Bisht J, Sankhyan N, Kaushal RK, Sharma RC, Grover N. Clinical profile of pediatric somatoform disorders. Indian Pediatr 2008;45:111-115.

34. Kamate M, Chetal V, Tonape V, Mahantshetti N, Hattiholi V. Central nervous system inflammatory demyelinating disorders of childhood. Ann Indian Acad Neurol 2010;13:289-292.

35. Oskoui M, Coutinho F, Dykeman J, Jette N, Pringsheim T. An update on the prevalence of cerebral palsy: a systematic review and meta-analysis. Dev Med Child Neurol 2013;55: 509-519.

36. Elsabbagh M, Divan G, Koh YJ, et al. Global prevalence of autism and other pervasive developmental disorders. Austism Res 2012;5:160-179. 
37. Sival DA, Pouwels ME, Brederode Van, et al. In children with Friedreich ataxia, muscle and ataxia parameters are associated. Dev Med Child Neurol 2011;53:529-534.

38. Di Prospero NA, Baker A, Jeffries N, Fischbeck KH. Neurological effects of high-dose idebenone in patients with Friedreich's ataxia: a randomized, placebo-controlled trial. Lancet Neurol 2007;6:878-886.

39. Lynch DR, Perlman SL, Meier T. A phase 3, doubleblind, placebo-controlled trial of idebenone in friedreich ataxia. Arch Neurol 2010;67:941-947.

40. Brandsema JF, Stephens D, Hartley J, Yoon G. Intermediatedose idebenone and quality of life in Friedreich ataxia. Pediatr Neurol 2010;42:338-342.

41. Zannolli R, Buoni S, Betti G, et al. A randomized trial of oral betamethasone to reduce ataxia symptoms in ataxia telangiectasia. Mov Disord 2012;27:1312-1316.

42. Nissenkorn A, Hassin-Baer S, Lerman SF, Levi YB, Tzadok M, Ben-Zeev B. Movement disorder in ataxia- telangiectasia: treatment with amantadine sulfate. J Child Neurol 2013;28:155-160.

43. Cernak K, Stevens V, Price R, Shumway-Cook A. Locomotor training using body-weight support on a treadmill in conjunction with ongoing physical therapy in a child with severe cerebellar ataxia. Phys Ther 2008;88:88-97.

44. Harris-Love MO, Siegel KL, Paul SM, Benson K. Rehabilitation management of Friedreich ataxia: lower extremity force-control variability and gait performance. Neurorehabil Neural Repair 2004;18:117-124.

45. Ilg W, Schatton C, Schicks J, Giese MA, Schöls L, Synofzik M. Video game-based coordinative training improves ataxia in children with degenerative ataxia. Neurology 2012;79:2056-2060.

46. López-Bastida J, Perestelo-Pérez L, Montón-Alvarez F, Serrano-Aguilar P. Social economic costs and healthrelated quality of life in patients with degenerative cerebellar ataxia in Spain. Mov Disord 2008;23:212-217. 